\title{
Control of Nonlinear Distributed Parameter Systems Based on Global Approximation
}

\author{
Chunyan Du ${ }^{1}$ and Guansheng Xing ${ }^{2}$ \\ ${ }^{1}$ School of Electrical Engineering and Automation, Tianjin University, Tianjin, China \\ ${ }^{2}$ School of Electrical Engineering and Automation, Hebei University of Technology, Tianjin, China \\ Correspondence should be addressed to Chunyan Du; ducy@tju.edu.cn
}

Received 6 June 2014; Accepted 24 June 2014; Published 14 August 2014

Academic Editor: Giuseppe Marino

Copyright (c) 2014 C. Du and G. Xing. This is an open access article distributed under the Creative Commons Attribution License, which permits unrestricted use, distribution, and reproduction in any medium, provided the original work is properly cited.

\begin{abstract}
We extend an iterative approximation method to nonlinear, distributed parameter systems given by partial differential and functional equations. The nonlinear system is approached by a sequence of linear time-varying systems, which globally converges in the limit to the original nonlinear systems considered. This allows many linear control techniques to be applied to nonlinear systems. Here we design a sliding mode controller for a nonlinear wave equation to demonstrate the effectiveness of this method.
\end{abstract}

\section{Introduction}

The control of finite-dimensional nonlinear system of the form

$$
\dot{x}(t)=A(x, N(x, \theta)) x(t)+B u
$$

has recently been studied via a sequence of linear timevarying (LTV) approximation of the form

$$
\begin{array}{r}
\dot{x}^{[i]}(t)=A\left(x^{[i-1]}, N\left(x^{[i-1]}, \theta\right)\right) x^{[i]}(t)+B\left(x^{[i-1]}(t)\right) u, \\
i=1,2,3,4, \ldots,
\end{array}
$$

where $N(x, \theta)$ is some nonlinear function defined over the interval $[t-\theta, t]$.

This iterative linear approximation method makes it convenient to control nonlinear systems via linear feedback control technique. It has been shown to be effective in sliding control [1] and optimal control [2]. The basic theory and convergence is presented in $[3,4]$.

However, many real systems are distributed parameter systems described by partial differential and functional equations. Comparing with the lumped parameter systems, it is more difficult to control these systems, especially for nonlinear distributed parameter systems. Different methods are applied to different kinds of nonlinear distributed parameter equations (PDE). The Galerkin's method is used to transfer nonlinear parabolic equations into nonlinear ordinary differential equations (ODE), which allows many control methods to be applied to nonlinear parabolic equations. In [5] ElFarra et al. develop feedback linearization method based on Lyapunov techniques. In [6] Wu and Li design a fuzzy observer-based controller based on T-S model of the ODE, while in $[7,8] \mathrm{Wu}$ and $\mathrm{Li}$ design linear model feedback controllers based on neural network approximation of the ODE. In [9] Deng et al. develop a spectral approximation method to distributed thermal processing, and a hybrid general regression $\mathrm{NN}$ is trained to be a nonlinear model of the original PDE, which also allows many control methods to be applied to this kind of nonlinear PDEs.

In this paper we will extend the above-mentioned iterative linear approximation theory to the nonlinear wave equation, which is a typical infinite-dimensional nonlinear $\mathrm{PDE}$ of the form

$$
\begin{array}{r}
\frac{\partial^{2} \phi}{\partial t^{2}}=\omega\left(1+r \phi^{2}\right) \frac{\partial^{2} \phi}{\partial x^{2}}+u, \\
x \in \Omega, \quad t \in(0, \tau),
\end{array}
$$

where $r$ and $\omega$ are constant and the wave frequency is a nonlinear function of $\phi$. And we design a sliding mode controller based on the approximation method. 
The nonlinear wave equation is transformed into a sequence of LTV approximations, each of which has an infinite-dimensional sliding surface which is time-varying. The limit of these surfaces gives an effective nonlinear sliding surface for the original system. And the limit of these sliding mode controllers will exponentially stabilizes the nonlinear wave equation.

Section 2 recalls the principles of the sliding mode control for finite-dimensional nonlinear systems based on LTV approximation method. Section 3 designs a sequence of LTV approximations for nonlinear wave equation and proves their convergence. Section 4 develops a sliding controller for the nonlinear wave equation based on LTV approximations. Sections 5 and 6 present the simulation result and the conclusion.

\section{Sliding Control for Finite-Dimensional Nonlinear Systems}

2.1. LTV Approximation of Finite-Dimensional Nonlinear Systems. For a nonlinear finite-dimensional system given by a functional differential equation

$$
\dot{x}(t)=A(x, N(x, \theta)) x(t),
$$

where $N(x, \theta)$ is some nonlinear function defined over the interval $[t-\theta, t]$, we can define a series LTV approximations as follows:

$$
\begin{gathered}
\dot{x}^{[0]}(t)=A\left(x_{0}, N\left(x_{0}, \theta\right)\right) x^{[0]}(t), \\
\dot{x}^{[i]}(t)=A\left(x^{[i-1]}(t), N\left(x^{[i-1]}(t), \theta\right)\right) x^{[i]}(t), \\
x^{[0]}(0)=x^{[i]}(0)=x_{0} \in R .
\end{gathered}
$$

The approximations of the form (5) are proved to be global convergent under a mild Lipschitz condition [4].

Theorem 1. Suppose that the nonlinear functional differential equation (4) has a unique solution on the interval $[t-\theta, t]$ and assume that the following condition holds:

$$
\begin{array}{r}
\left\|A\left(x_{1}, N\left(x_{1}, q\right)\right)-A\left(x_{2}, N\left(x_{2}, q\right)\right)\right\| \leq \alpha(K)\left\|x_{1}-x_{2}\right\|, \\
\text { for } x_{1}, x_{2} \in B\left(K ; x_{0}\right),
\end{array}
$$

where $B\left(K ; x_{0}\right) \in R^{N}$ is a ball of radius $K$ centered at $x_{0}$ and $R$ is a constant related to $K$. Then the sequence of $x^{[i]}(t)$ defined in (5) converges uniformly on $[t-\theta, t]$.

This method makes it possible to control nonlinear system in form of (1) using linear feedback control technique, such as sliding control and optimal control.
2.2. Sliding Control for Nonlinear Systems Based on LTV Approximation. Apply the approximation technique for system (1); the following sequence of LTV can be obtained:

$$
\begin{gathered}
\dot{x}^{[0]}(t)=A\left(x_{0}, N\left(x_{0}, \theta\right)\right) x^{[0]}(t)+B u^{[0]}(t), \\
\dot{x}^{[i]}(t)=A\left(x^{[i-1]}(t), N\left(x^{[i-1]}(t), \theta\right)\right) x^{[i]}(t)+B u^{[i]}(t), \\
x^{[0]}(0)=x^{[i]}(0)=x_{0} \in R .
\end{gathered}
$$

For each of these LTV equations a sliding mode controller can be designed as follows:

$$
\begin{gathered}
\sigma^{[i]}(t)=0, \\
\dot{\sigma}^{[i]}(t)=-\operatorname{sign}\left(\sigma^{[i]}(t)\right),
\end{gathered}
$$

where $\sigma^{[i]}(t)=0$ is a time-varying sliding surface. Once the system hits the surface, the reduced order system will remain stable. The system is driven into the sliding surface by setting derivative of $\sigma^{[i]}$ equal to minus sign of $\sigma^{[i]}$. Details on how to choose the sliding surface and get the control input for LTV systems can be found in [10].

Thus we can get a series of sliding mode controller $u^{[i]}(t)$ for the LTV systems (7). It is proved that under a mild condition, $u^{[i]}(t)$ converge as $i \rightarrow \infty$. The limit of the sliding surfaces gives an effective nonlinear sliding surface for the system, and the limit of $u^{[i]}(t)$ will exponentially stabilize the original nonlinear equation (1) [3].

\section{Linear Approximation of Nonlinear Wave Equation}

In this section, we extend the iterative approximation method to nonlinear, distributed parameter systems given by partial differential and functional equations. As an example, we design a sequence of iterative LTV distributed parameter systems to approximate the nonlinear wave equation and prove their convergence.

First, we transfer (3) to a standard style. Define a variable as follows:

$$
\psi(x, t)=\frac{\partial \phi}{\partial t} .
$$

Thus (3) could be written as

$$
\begin{gathered}
\frac{\partial \phi}{\partial t}=\psi, \\
\frac{\partial \psi}{\partial t}=\omega\left(1+r \phi^{2}\right) \frac{\partial^{2} \phi}{\partial x^{2}} .
\end{gathered}
$$


From (5), we can get a sequence of LTV approximation system of (10) as follows:

$$
\begin{gathered}
\frac{\partial \phi^{[i]}}{\partial t}=\psi^{[i]}, \\
\frac{\partial \psi^{[i]}}{\partial t}=\omega\left[1+r\left(\phi^{[i-1]}\right)^{2}\right] \frac{\partial^{2} \phi^{[i]}}{\partial x^{2}}, \\
i=1,2, \ldots, N,
\end{gathered}
$$

where the initial values of each system are defined by the initial value function $f(x)$ of (3) as

$$
\begin{gathered}
\phi^{[i]}(x, 0)=f(x), \quad x \in(0, l) ; \\
\psi^{[i]}(x, 0)=0, \quad x \in(0, l) ; \\
\phi^{[0]}(x, t)=f(x), \quad t \in(0, \tau) ; \\
\psi^{[0]}(x, t)=0, \quad t \in(0, \tau)
\end{gathered}
$$

and the first LTV approximation system (when $i=1$ ) is defined as

$$
\begin{gathered}
\frac{\partial \phi^{[1]}}{\partial t}=\psi^{[1]}, \\
\frac{\partial \psi^{[1]}}{\partial t}=\omega\left[1+r\left(\phi^{[0]}\right)^{2}\right] \frac{\partial^{2} \phi^{[1]}}{\partial x^{2}} .
\end{gathered}
$$

Obviously, each LTV system of (11) is a distributed parameter system and has a unique solution. Here we will prove that this sequence of systems will converge to the original nonlinear system (10).
Proof. Firstly, we use a sequence of finite-dimensional difference equations to approximate equation (11).

For $\phi^{[i]}(x, t)$ and $\psi^{[i]}(x, t)(x \in[0, l])$, define

$$
\begin{array}{r}
\phi_{j}^{[i]}(t)=\phi^{[i]}\left(\frac{j l}{M}, t\right), \\
\psi_{j}^{[i]}(t)=\psi^{[i]}\left(\frac{j l}{M}, t\right), \\
j=1,2, \ldots, M
\end{array}
$$

with initial value

$$
\begin{gathered}
\left(\phi_{1}^{[i]}(0), \phi_{2}^{[i]}(0), \ldots, \phi_{M}^{[i]}(0)\right) \\
=\left(f\left(\frac{l}{M}\right), f\left(\frac{2 l}{M}\right), \ldots, f(l)\right), \\
\left(\psi_{1}^{[i]}(0), \psi_{2}^{[i]}(0), \ldots, \psi_{M}^{[i]}(0)\right)=(0,0, \ldots, 0) .
\end{gathered}
$$

Suppose that when $M \rightarrow \infty$, (11) could be approximated by the following finite-dimensional system [11]:

$$
\left[\begin{array}{l}
\dot{\boldsymbol{\Phi}}^{[i]}(t) \\
\dot{\Psi}^{[i]}(t)
\end{array}\right]=A^{[i-1]}\left[\begin{array}{l}
\boldsymbol{\Phi}^{[i]}(t) \\
\boldsymbol{\Psi}^{[i]}(t)
\end{array}\right],
$$

where

$$
\begin{aligned}
\boldsymbol{\Phi}^{[i]}(t) & =\left[\phi_{1}^{[i]}(t), \phi_{2}^{[i]}(t), \ldots, \phi_{M}^{[i]}(t)\right]^{T}, \\
\Psi^{[i]}(t) & =\left[\psi_{1}^{[i]}(t), \psi_{2}^{[i]}(t), \ldots, \psi_{M}^{[i]}(t)\right]^{T},
\end{aligned}
$$

and $A^{[i-1]}$ is defined as follows:

$$
A^{[i-1]}=\left[\begin{array}{cccccccc}
0 & 0 & \cdots & 0 & 1 & 0 & \cdots & 0 \\
0 & 0 & \cdots & 0 & 0 & 1 & \cdots & 0 \\
\vdots & \vdots & \vdots & \vdots & \vdots & \vdots & \vdots & \vdots \\
0 & 0 & \cdots & 0 & 0 & 0 & \cdots & 1 \\
\frac{-2 \omega\left(1+\phi_{1}^{[i-1]}\right)^{2}}{(l / M)^{2}} & \frac{\omega\left(1+\phi_{1}^{[i-1]}\right)^{2}}{(l / M)^{2}} & \cdots & 0 & 0 & 0 & \cdots & 0 \\
\frac{\omega\left(1+\phi_{2}^{[i-1]}\right)^{2}}{(l / M)^{2}} & \frac{-2 \omega\left(1+\phi_{2}^{[i-1]}\right)^{2}}{(l / M)^{2}} & \cdots & 0 & 0 & 0 & \cdots & 0 \\
\vdots & \vdots & \vdots & \vdots & \vdots & \vdots & \vdots & \vdots \\
0 & 0 & \cdots & \frac{-2 \omega\left(1+\phi_{M}^{[i-1]}\right)^{2}}{(l / M)^{2}} & 0 & 0 & \cdots & 0
\end{array}\right] .
$$

Secondly, we use a sequence of finite-dimensional difference equations to approximate equation (10); define

$$
\begin{aligned}
\phi_{j}(t) & =\phi\left(\frac{j l}{M}, t\right), \\
\psi_{j}(t) & =\psi\left(\frac{j l}{M}, t\right), \\
j & =1,2, \ldots, M
\end{aligned}
$$

$$
\begin{gathered}
\left(\phi_{1}(0), \phi_{2}(0), \ldots, \phi_{M}(0)\right)=\left(f\left(\frac{l}{M}\right), f\left(\frac{2 l}{M}\right), \ldots, f(l)\right), \\
\left(\psi_{1}(0), \psi_{2}(0), \ldots, \psi_{M}(0)\right)=(0,0, \ldots, 0) .
\end{gathered}
$$


Suppose that when $M \rightarrow \infty$, (10) could be approximated by the following finite-dimensional system:

$$
\left[\begin{array}{l}
\dot{\boldsymbol{\Phi}}(t) \\
\dot{\boldsymbol{\Psi}}(t)
\end{array}\right]=A\left[\begin{array}{l}
\boldsymbol{\Phi}(t) \\
\boldsymbol{\Psi}(t)
\end{array}\right]
$$

$$
A=\left[\begin{array}{cccccccc}
0 & 0 & \cdots & 0 & 1 & 0 & \cdots & 0 \\
0 & 0 & \cdots & 0 & 0 & 1 & \cdots & 0 \\
\vdots & \vdots & \vdots & \vdots & \vdots & \vdots & \vdots & \vdots \\
0 & 0 & \cdots & 0 & 0 & 0 & \cdots & 1 \\
\frac{-2 \omega\left(1+\phi_{1}\right)^{2}}{(l / M)^{2}} & \frac{\omega\left(1+\phi_{1}\right)^{2}}{(l / M)^{2}} & \cdots & 0 & 0 & 0 & \cdots & 0 \\
\frac{\omega\left(1+\phi_{2}\right)^{2}}{(l / M)^{2}} & \frac{-2 \omega\left(1+\phi_{2}\right)^{2}}{(l / M)^{2}} & \cdots & 0 & 0 & 0 & \cdots & 0 \\
\vdots & \vdots & \vdots & \vdots & \vdots & \vdots & \vdots & \vdots \\
0 & 0 & \cdots & \frac{-2 \omega\left(1+\phi_{M}\right)^{2}}{(l / M)^{2}} & 0 & 0 & \cdots & 0
\end{array}\right] .
$$

Obviously, matrix A satisfies local Lipschitz condition. From Theorem 1 we could deduce that when $i \rightarrow \infty$, the liner time-varying systems (16) will converge uniformly to the nonlinear system (21).

Suppose, at the same time, that when $M \rightarrow \infty$, the distributed parameter system (11) and (10) will be approximated by difference approximation equations (16) and (21), separately. Thus we could know that (11) will eventually converge to (10).

Conclusion. When $i \rightarrow \infty$, the linear time-varying distributed parameter systems (11) will converge uniformly to the nonlinear distributed parameter system (10).

Remark. This approximation method could be extended to other nonlinear distributed parameter systems, as far as the systems could be approximated by difference equations and satisfy local Lipschitz condition.

\section{Sliding Control of Nonlinear Wave Equation}

In this section, we design a sliding mode controller for the nonlinear wave equation based on the approximation method.

Consider the control problem

$$
\begin{array}{r}
\frac{\partial^{2} \phi}{\partial t^{2}}=\omega\left(1+r \phi^{2}\right) \frac{\partial^{2} \phi}{\partial x^{2}}+u(x, t), \\
x \in[0, l], \quad t \in(0, \tau) .
\end{array}
$$

Here we will design a controller to stabilize this system. where

$$
\begin{aligned}
\boldsymbol{\Phi}(t) & =\left[\phi_{1}(t), \phi_{2}(t), \ldots, \phi_{M}(t)\right]^{T}, \\
\Psi(t) & =\left[\psi_{1}(t), \psi_{2}(t), \ldots, \psi_{M}(t)\right]^{T},
\end{aligned}
$$

and $A$ is defined as follows:

As in Section 3, system (24) could be approximated by LTV distributed parameter equation

$$
\begin{gathered}
\frac{\partial \phi^{[i]}}{\partial t}=\psi^{[i]} \\
\frac{\partial \psi^{[i]}}{\partial t}=\omega\left[1+r\left(\phi^{[i-1]}\right)^{2}\right] \frac{\partial^{2} \phi^{[i]}}{\partial x^{2}}+u^{[i]}(x, t) .
\end{gathered}
$$

Thus we could design a series of sliding mode surfaces and controllers for the above linear problem. Under the local Lipschitz condition, when $i \rightarrow \infty$, the sliding mode surfaces and controllers are convergent. The limit of the surfaces is an effective sliding mode surface of the original nonlinear system, and the limit of the sliding mode controllers could eventually stabilize the nonlinear system. Here we notice that both of the sliding mode surface and the controller are distributed parameter system.

We choose an infinite-dimensional time-varying sliding surface for each system as

$$
\sigma^{[i]}(x, t)=\psi^{[i]}-\omega\left[1+r\left(\phi^{[i-1]}\right)^{2}\right] \frac{\partial^{2} \phi^{[i]}}{\partial x^{2}} .
$$

When the system is on the surface, that is $\sigma=0$, we can get the reduced order system

$$
\psi^{[i]}=\omega\left[1+r\left(\phi^{[i-1]}\right)^{2}\right] \frac{\partial^{2} \phi^{[i]}}{\partial x^{2}} .
$$

Notice that system (27) is a time-varying heating equation, which is always stable [12]. That means once the system hits the sliding surface, it will remain stable.

To drive the system to this surface, set derivative of $\sigma^{[i]}$ equal to minus sign of $\sigma^{[i]}$ as follows:

$$
\dot{\sigma}^{[i]}(x, t)=-\operatorname{sign}\left(\sigma^{[i]}(x, t)\right) .
$$


By substituting (28) into (26), we get

$$
u^{[i]}=-\operatorname{sign}\left(\sigma^{[i]}\right)+2 r w \dot{\phi}^{[i-1]} \frac{\partial^{2} \phi^{[i]}}{\partial x^{2}} .
$$

\section{Simulation Result}

We approximate each system of (11) by a definite-dimensional approximation. For

$$
\phi^{[i]}(x, t), \quad x \in[0, l],
$$

we could write

$$
\phi_{j}^{[i]}(x, t)=\phi^{[i]}\left(\frac{j l}{N}, t\right), \quad j=1,2,3, \ldots, N .
$$

Then the LTV approximation of nonlinear wave equation (11) can be written as

$$
\begin{gathered}
\phi_{j}^{[i]}(t)=\phi^{[i]}\left(\frac{j l}{M}, t\right), \\
\psi_{j}^{[i]}(t)=\psi^{[i]}\left(\frac{j l}{M}, t\right), \quad j=1,2, \ldots, M .
\end{gathered}
$$

And the systems (26) can be written as

$$
\begin{gathered}
\dot{\phi}_{j}^{[i]}=\psi_{j}^{[i]}, \\
\dot{\psi}_{j}^{[i]}=\frac{\omega\left[1+\left(r \phi_{j}^{[i-1]}\right)^{2}\right]\left(-2 \phi_{j}^{[i]}+\phi_{j-1}^{[i]}+\phi_{j+1}^{[i]}\right)}{(l / M)^{2}}+u_{j}^{[i]}
\end{gathered}
$$

with control input

$$
u_{j}^{[i]}=-\operatorname{sign}\left(\sigma_{j}^{[i]}\right)+\frac{2 r \omega \dot{\phi}_{j}^{[i-1]}\left(-2 \phi_{j}^{[i]}+\phi_{j-1}^{[i]}+\phi_{j+1}^{[i]}\right)}{(l / M)^{2}},
$$

where the initial values

$$
\phi_{j}^{[i]}(0)=\phi_{j_{0}}, \quad \psi_{j}^{[i]}(0)=\psi_{j_{0}}
$$

and the boundary condition

$$
\phi_{0}^{[i]}(0)=0, \quad \phi_{M}^{[i]}(0)=0 .
$$

The simulation is performed in MATLAB by using Euler numerical integration technique. The parameters are

$$
\begin{gathered}
w=0.4, \quad r=0.5, \quad l=1, \\
N=100, \quad t \in[0,1] s, \\
\phi_{j_{0}}=\left\{\begin{array}{cc}
\frac{j}{50}, & \text { if } 1 \leq j \leq 50, \\
1-\frac{(j-50)}{50}, & \text { if } 50<j \leq 100, \\
\psi_{j_{0}}=0 .
\end{array}\right.
\end{gathered}
$$

Figure 1 gives the approximation error of (11), where the red part denotes the error when $i=2$, and the blue part denotes the error when $i=4$. As is shown in the figure, the approximation error decreases as iteration time increases.

Figure 2 gives the control result when applying $u^{[4]}$ to the original nonlinear wave system. As is shown in the figure, the system is stabilized.

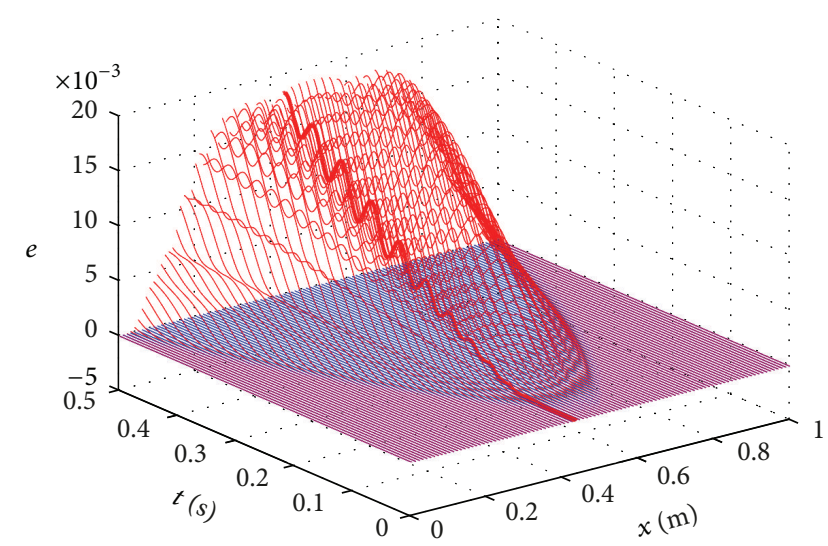

Figure 1: Approximation error.

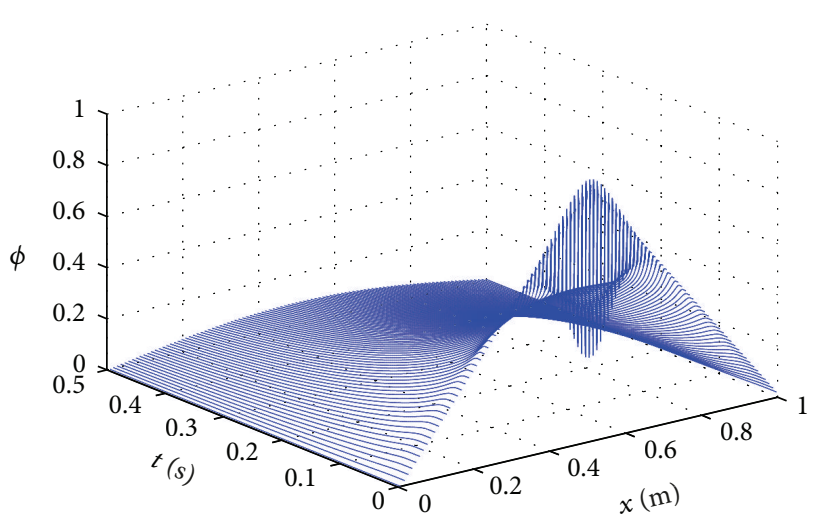

FIGURE 2: Control result.

\section{Conclusion}

In this paper, we extend a recently introduced approximation method to nonlinear distributed parameter system and design a sliding mode controller for a nonlinear wave equation. The nonlinear wave equation is replaced by a sequence of LTV systems, which are proved to be globally convergent under certain conditions. An infinite-dimensional LTV sliding surface is designed for each of the LTV approximation. The limit of these surfaces gives an effective nonlinear sliding surface for the original system. And the limit of these sliding mode controllers exponentially stabilizes the nonlinear wave equation. The control result shows the effectiveness of this method.

\section{Conflict of Interests}

The authors declare that there is no conflict of interests regarding the publication of this paper.

\section{Acknowledgments}

This work was partially supported by the National Nature Science Foundation of China (no. 61271321), Natural Science Foundation of Hebei Province (no. F2013202101), and Science 
and Technology Support Program of Hebei Province (no. 13211827).

\section{References}

[1] M. Tomas-Rodriguez, S. P. Banks, and M. U. Salamci, "Sliding mode control for nonlinear systems: an iterative approach," in Proceedings of the 45th IEEE Conference on Decision and Control, pp. 4963-4968, San Diego, Calif, USA, December 2006.

[2] T. Çimen and S. P. Banks, "Global optimal feedback control for general nonlinear systems with nonquadratic performance criteria," Systems and Control Letters, vol. 53, no. 5, pp. 327-346, 2004.

[3] M. Tomas-Rodriguez and S. P. Banks, "Linear approximations to nonlinear dynamical systems with applications to stability and spectral theory," IMA Journal of Mathematical Control and Information, vol. 20, no. 1, pp. 89-103, 2003.

[4] C. Du, X. Xu, S. P. Banks, and A. Wu, "Control of nonlinear functional differential equations," Nonlinear Analysis: Theory, Methods \& Applications, vol. 71, no. 12, pp. e1850-e1857, 2009.

[5] N. H. El-Farra, A. Armaou, and P. D. Christofides, "Analysis and control of parabolic PDE systems with input constraints," Automatica, vol. 39, no. 4, pp. 715-725, 2003.

[6] H.-N. Wu and H.-X. Li, " $H_{\infty}$ fuzzy observer-based control for a class of nonlinear distributed parameter systems with control constraints," IEEE Transactions on Fuzzy Systems, vol. 16, no. 2, pp. 502-516, 2008.

[7] H.-N. Wu and H. Li, "A Galerkin/neural-network-based design of guaranteed cost control for nonlinear distributed parameter systems," IEEE Transactions on Neural Networks, vol. 19, no. 5, pp. 795-807, 2008.

[8] H.-N. Wu and H.-X. Li, "Adaptive neural control design for nonlinear distributed parameter systems with persistent bounded disturbances," IEEE Transactions on Neural Networks, vol. 20, no. 10, pp. 1630-1644, 2009.

[9] H. Deng, H. Li, and G. Chen, "Spectral-approximation-based intelligent modeling for distributed thermal processes," IEEE Transactions on Control Systems Technology, vol. 13, no. 5, pp. 686-700, 2005.

[10] H. Dallali, Sliding Mode Control of Nonlinear System [M.S. thesis in Control Systems], University of Sheffield, 2007.

[11] Z. Wensheng, Finite Difference Methods for Partial Differential Equations in Science Computation, China Higher Education Press, Beijing, China, 2006.

[12] W. Mingxin, Equations of Mathematical Physics, Tsinghua University Press, Beijing, China, 2009. 


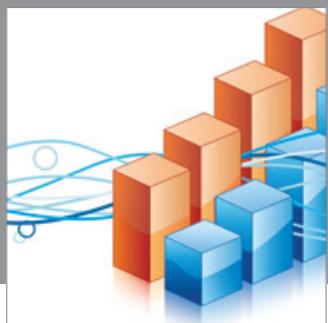

Advances in

Operations Research

mansans

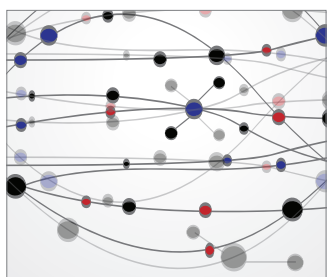

The Scientific World Journal
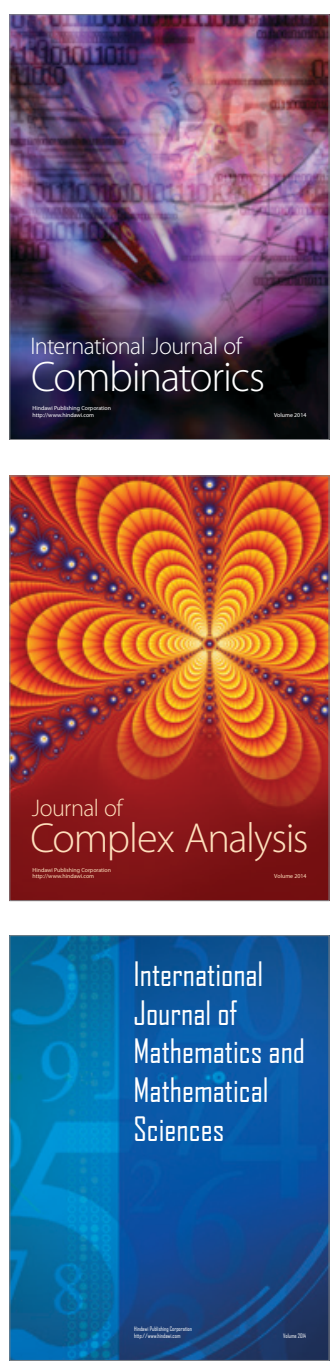
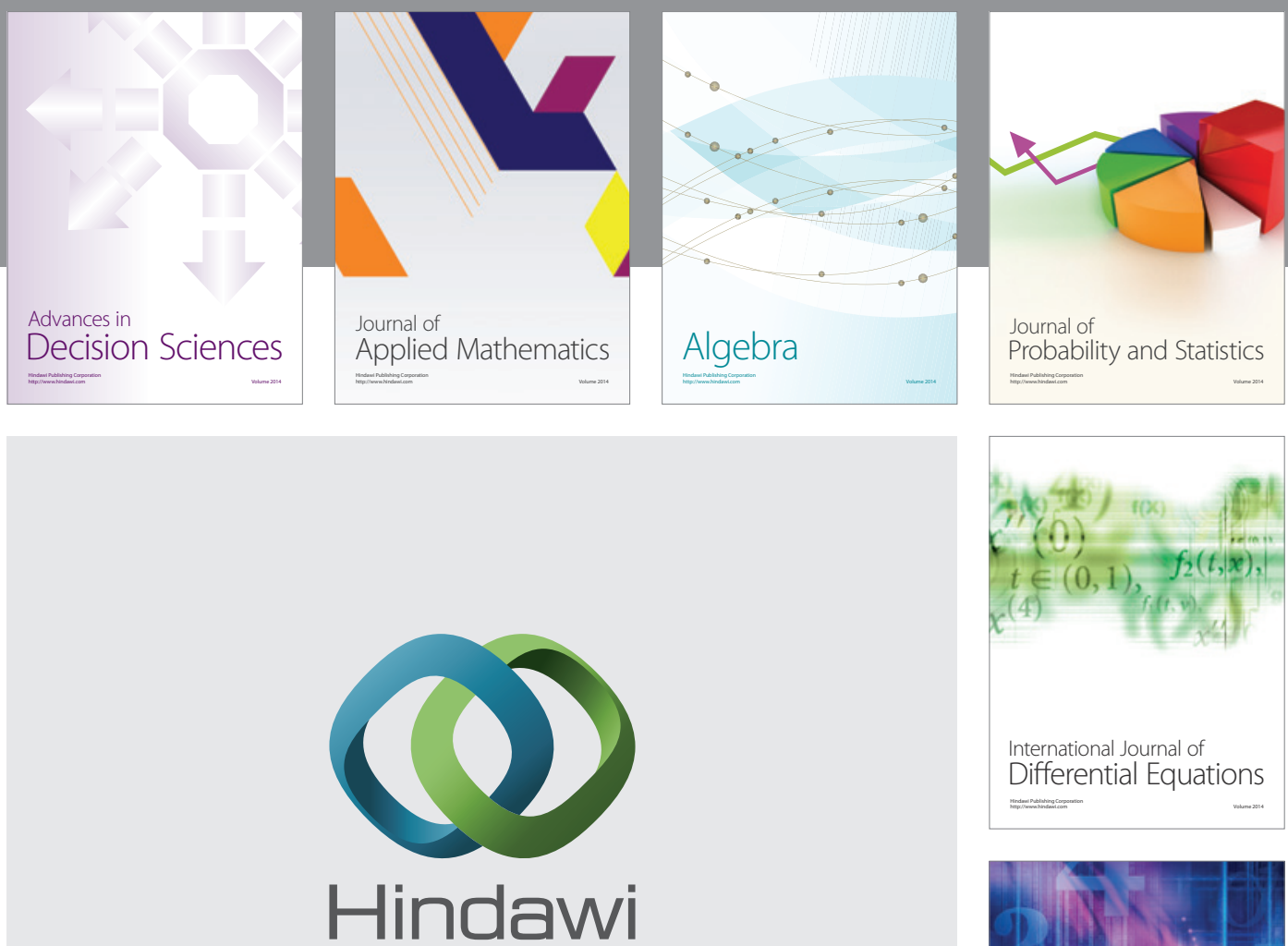

Submit your manuscripts at http://www.hindawi.com
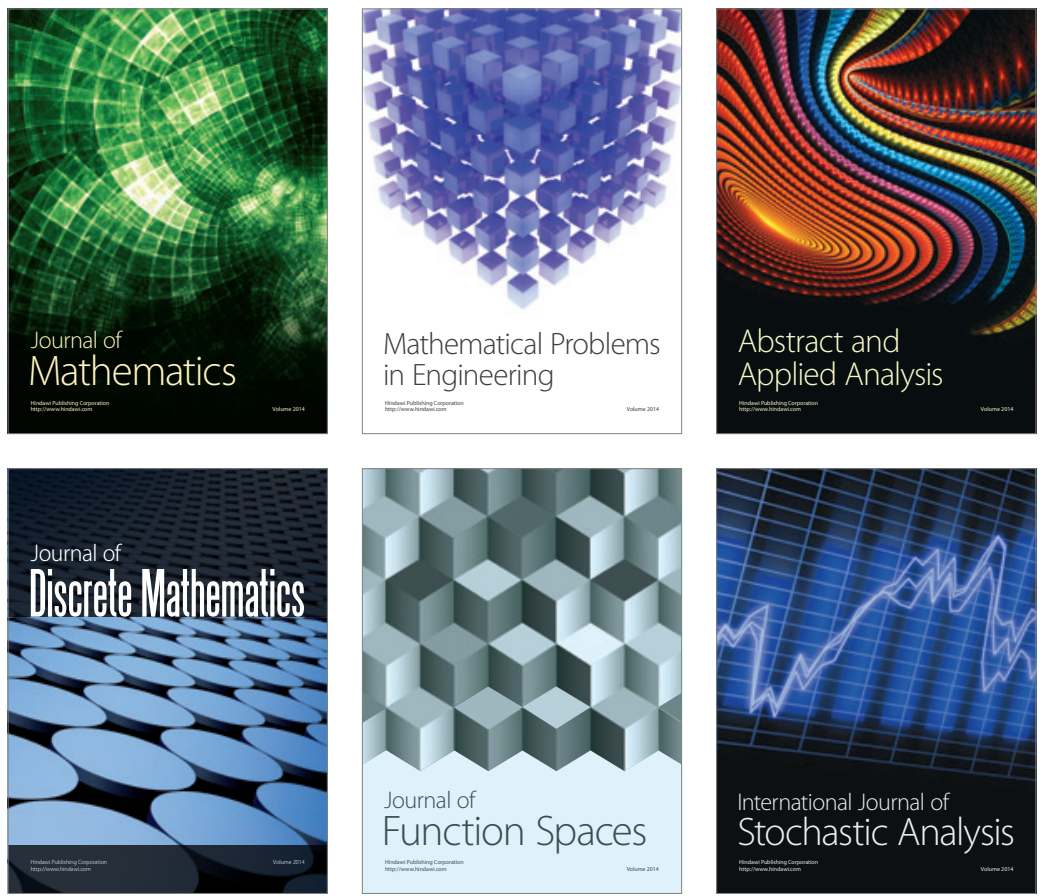

Journal of

Function Spaces

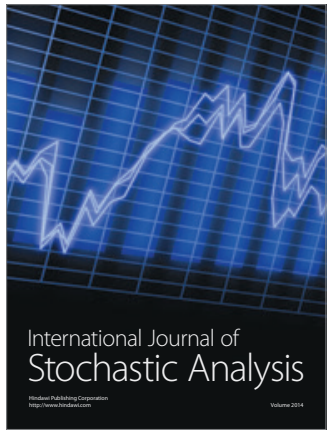

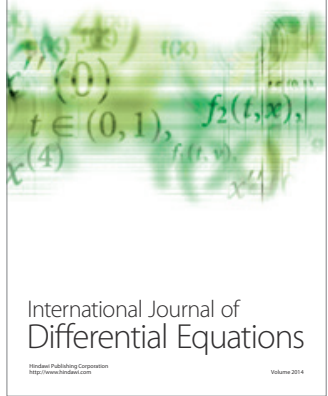
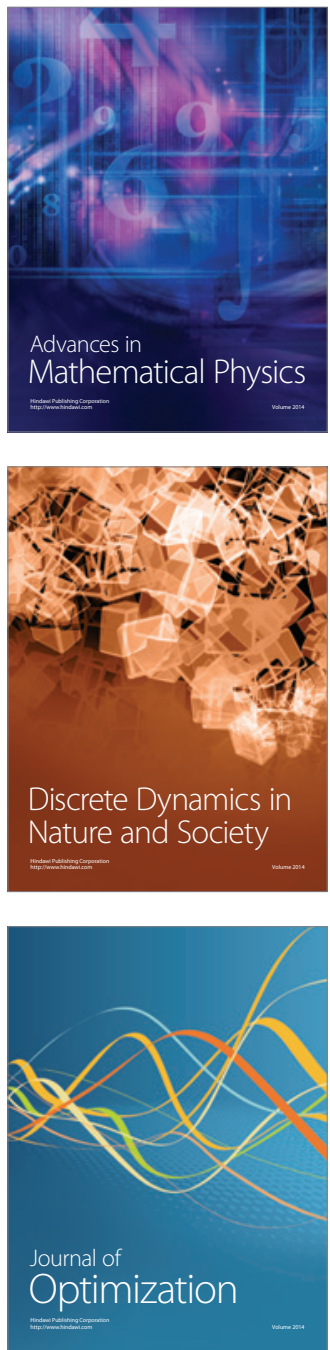